\title{
Corpus
}

\section{La dislocation en italien : une étude sur corpus}

\section{Sandra Augendre}

\section{(2) OpenEdition}

Journals

Édition électronique

URL : http://journals.openedition.org/corpus/1917

DOI : 10.4000/corpus. 1917

ISSN : 1765-3126

\section{Éditeur}

Bases; corpus et langage - UMR 6039

\section{Édition imprimée}

Date de publication : 1 novembre 2010

Pagination : 221-244

ISSN : 1638-9808

\section{Référence électronique}

Sandra Augendre, "La dislocation en italien : une étude sur corpus », Corpus [En ligne], 9 | 2010, mis en ligne le 14 juillet 2011, consulté le 08 septembre 2020. URL : http://journals.openedition.org/corpus/ 1917 ; DOI : https://doi.org/10.4000/corpus. 1917 


\title{
La dislocation en italien : une étude sur corpus
}

\author{
Sandra AUGENDRE \\ Université Michel de Montaigne Bordeaux 3 \\ Università degli Studi di Cagliari
}

\section{Introduction}

Classée parmi les structures dites «marquées » dans la grammaire italienne de référence, La Grande grammatica italiana di consultazione (Renzi, Salvi et Cardinaletti, 1988), la dislocation est le prototype de la construction productive à l'oral comme à l'écrit et perçue comme aussi naturelle qu'une structure alternative dans bon nombre de contextes et situations. Après une présentation des spécificités de la structure en italien, l'article se propose d'en étudier la réalisation et la distribution effective sur la base d'un corpus écrit de 200 occurrences de dislocation.

\section{La dislocation en italien}

Avant d'introduire le corpus que nous avons constitué et son analyse, nous allons commencer par présenter les propriétés formelles et informationnelles de la dislocation ainsi que les principales hypothèses et travaux déjà proposés sur la question.

\subsection{La dislocation parmi les structures à détachement}

La dislocation est une construction syntaxique dans laquelle un syntagme est « isolé » du reste de l'énoncé. Cependant, ce n'est pas la seule structure qui implique une zone de détachement :

- On parle de dislocation ${ }^{1}$ quand un syntagme est détaché avec ses marques fonctionnelles (préposition pour les

1 Dans la littérature sur la question, cette structure syntaxique se voit attribuer différentes dénominations, dont voici quelques exemples: «clitic topic » (Cinque, 1991), « dislocation construite» (Blasco-Dulbecco, 1999), « dislocation liée » (Gerdes et Kahane, 2006).

Corpus $\mathrm{n}^{\circ} 9$ 《La syntaxe de corpus / Corpus Syntax » (2010), 221-244 


\section{S. AUGENDRE}

arguments indirects) et qu'il est (normalement) associé à une reprise clitique au sein de l'énoncé.

(1) A Parigi, non ci vado mai.

À Paris, nég. y vais(1sg.) jamais.

«À Paris, je n'y vais jamais. »

Précisons d'ores et déjà que dans le cadre d'une dislocation du sujet, nous considérons qu'à défaut de pronom clitique sujet en italien, c'est la désinence verbale qui joue ce rôle anaphorique.

- On parle de thème détaché (TD) ${ }^{2}$ quand le syntagme est réalisé sans ses marques fonctionnelles et qu'il est repris dans l'énoncé par un pronom clitique ou une "proforme » (Ferrari, 2003 : 148), tel qu'un pronom tonique ou un syntagme plein (ex. 2).

(2) Piero, non parlo più con lui/quest'idiota da molto.

Pierre nég. parle(1sg.) plus avec lui/cet idiot depuis longtemps.

«Pierre, je ne parle plus avec lui/cet idiot depuis longtemps. »

Si les deux structures précédentes sont directement identifiables dans le cadre du détachement d'un SP, ce n'est pas le cas pour un objet direct. Afin de distinguer dislocation et TD quand la reprise de l'objet est clitique, Stammerjohann (1986: 265) propose de s'appuyer sur des facteurs prosodiques (pause « en général » plus importante quand il s'agit d'un TL) et pragmatiques (un élément disloqué est "normalement» donné). Pour notre part, nous préférons nous baser sur l'aspect syntaxique de l'énoncé et parler de dislocation de l'objet quand la reprise est clitique et de TD quand ce n'est pas le cas.

- On parle de thème libre (TL) quand le syntagme est détaché sans ses marques fonctionnelles et qu'il n'est pas repris au sein de l'énoncé.

(3) Le lingue, non capisco niente.

Les langues nég. comprends(1sg.) rien.

«Les langues, je ne comprends rien. »

2 Cette construction est également appelée: «nominativus pendens» (maintien des termes latins), «tema sospeso », "hanging topic» (Cinque, 1991), «dislocation non construite (Blasco-Dulbecco, 1999) ou encore « dislocation libre» (Gerdes et Kahane, 2006). 
Ce dernier cas est limité aux syntagmes introduits par une préposition et ne concerne ni le sujet, puisque ce dernier est toujours «repris » par la désinence verbale, ni l'objet direct, argument qui, nous y reviendrons, perd son statut thématique et passe du rôle de support à celui d'apport informationnel en l'absence de reprise pronominale.

Décrits à juste titre par Berruto (1985) comme différentes manifestations d'un même phénomène, ces trois types de détachements se distinguent et par leur degré décroissant de transparence syntaxique (dislocation > thème détaché > thème libre) et par le marquage rectionnel (double dans une dislocation, porté par la reprise pour un TD et nul ou plutôt «à construire » pour un TL). Contrairement aux TD et TL, qui introduisent un référent «nu» (identification > relation), le maintien des marques fonctionnelles dans la dislocation fait que la relation entre le syntagme disloqué et l'action ou l'évènement décrits dans le reste de l'énoncé est directement accessible et prévaut sur la visée identificatoire (relation > identification).

\subsection{Spécificités de la dislocation en italien}

Après quelques remarques préliminaires sur la terminologie employée, nous allons simplement présenter les principales caractéristiques de la dislocation en italien.

\subsubsection{Remarques préliminaires}

Avant toute chose, il nous semble nécessaire de consacrer quelques lignes au terme «dislocation » et aux qualificatifs «à gauche » et «à droite $»^{3}$ qui lui sont associés en fonction du placement du syntagme avant ou après le «bloc principal ».

L'appellation «dislocation» laisse entendre qu'il y a déplacement ou modification à partir d'une structure première (SVO pour l'italien). Cependant, le terme ne doit être vu que comme une simple dénomination, toute structuration de l'énoncé n'étant pas reliée à une structure non marquée ou canonique ${ }^{4}$

3 Dorénavant, nous utiliserons DG pour dislocation/disloqué à gauche et DD pour dislocation / disloqué à droite.

4 Nous renvoyons à Augendre (2008) pour plus de détails sur les notions d'ordre marqué et non marqué en italien. 


\section{S. AUGENDRE}

mais à un besoin communicatif, ce que souligne à juste titre Sobrero (1993 : 425-526, note 21) :

\section{Si tratta della sistemazione degli elementi in un certo ordine, che a seconda della funczione pragmatica prevalente può prevedere una collo- cazione dell'elemento prima o dopo il verbo ${ }^{5}$.}

La seconde remarque est la suivante : malgré le fait que la dislocation soit particulièrement productive dans la dimension orale de la langue, c'est en lien avec la linéarité du signifiant à l'écrit qu'est qualifié le type de détachement (à gauche $v s$ à droite). Même si ce n'est pas l'objet de ce présent travail, parler de dislocation à l'initiale ou en finale permettrait d'englober les deux dimensions.

Enfin, il est à noter que lorsque nous parlons de dislocation du sujet, du COD, du COI... il faut entendre que l'élément détaché se verrait attribuer cette fonction s'il n'était pas isolé du reste de la phrase et s'il n'y avait pas de pronom référentiellement et fonctionnellement identique dans l'énoncé mais pas qu'il assume cette fonction lorsqu'il est disloqué. Prenons l'exemple de la dislocation d'un pronom personnel afin d'illustrer cette distinction :

(4a) Lui, (io) lo detesto.

(4b) Lui, je le déteste.

Dans l'énoncé italien, comme dans son correspondant français, ce n'est pas le pronom tonique «lui / lui » qui est COD mais le pronom clitique «le / lo». Les deux formes pronominales sont complémentaires : la tonique est la seule à pouvoir être détachée et la clitique est celle qui assume la fonction d'objet au sein de l'énoncé. Tout comme le terme «dislocation », dont nous avons vu qu'il amenait l'idée de transformation syntaxique, le fait d'attribuer une fonction à un syntagme qui se trouve hors énoncé prend implicitement comme point de référence une structure de base.

5 Trad. : «Il s'agit de la disposition des éléments dans un certain ordre qui, par rapport à la fonction pragmatique prédominante, peut prévoir le placement d'un élément avant ou après le verbe ». 


\subsubsection{Caractéristiques syntaxiques}

Par dislocation, on entend communément qu'un syntagme, accompagné de ses marques fonctionnelles, s'il en a, est détaché, extraposé ou isolé syntaxiquement et prosodiquement ${ }^{6}$ du reste de la phrase tout en y étant repris sous la forme d'un pronom clitique.

En italien, cette définition ne vaudrait pas dans les deux cas exposés ci-dessous :

1) Il n'y a pas de pronom clitique sujet en italien, ce qui rendrait «invisible» ou inexistante la dislocation du premier argument.

2) La reprise pronominale est obligatoire pour un COD disloqué, mais optionnelle pour tout autre élément détaché.

Sur le cas du sujet, contrairement au point de vue adopté dans la plupart des études sur la question (Duranti et Ochs, 1979 ; Renzi, Salvi, Cardinaletti, 1988 ; Rossi, 2005...), nous soutenons l'idée que la dislocation du sujet (DS) existe en italien et ce malgré le manque de pronom sujet clitique dans cette langue. Si dans la Grande grammatica italiana di consultazione (1988) ou chez Ferrari (2003), il est postulé qu'il n'y a DS qu'à partir du moment où le sujet est séparé du verbe par d'autres constituants, d'autres linguistes comme Graffi (1994) ne nient pas l'existence de la DS mais notent simplement que l'énoncé n'en fournit aucun indice visible. En effet, un sujet disloqué constituant, selon les termes de Bonvino (2005: 135), «un rappel lexical et référentiel d'un sujet exprimé par la seule morphologie verbale», la syntaxe ne permet effectivement pas de dire si un premier argument est oui ou non disloqué. Cependant, il nous semble évident que ce sont les niveaux prosodique et informationnel qui permettent de l'attester. D'ailleurs, le travail sur corpus proposé par Bonvino (2005) sur le sujet postverbal va dans ce sens, au moins pour ce qui est de la DD de cet argument ${ }^{7}$.

6 Le corpus sur lequel s'appuie ce travail étant écrit, nous ne traiterons pas les spécificités prosodiques de la dislocation.

7 Dans son livre, Bonvino (2005 : 115-116) distingue les énoncés VS et V,S en utilisant l'opposition sujet attaché $v s$ sujet non attaché. 


\section{S. AUGENDRE}

Concernant la reprise clitique, il semble que sa présence soit globalement plus qu'une forte tendance : dans le corpus de Berruto (1985), moins de $15 \%$ des cas de dislocation ne présentent pas de clitique (et/ou de marque casuelle) et dans le LIP (De Mauro et al.: 1993), la reprise est présente dans la quasi-totalité des cas selon Frascarelli (2003). Cependant, les données de notre corpus vont dans le sens d'une reprise nécessaire pour un COD et plutôt accessoire pour un SP (thèse également défendue par Sobrero : 1993). Attardons-nous sur le caractère obligatoire de la reprise dans le cas de la dislocation d'un COD. En tant qu'argument introduit directement, ce dernier souffre d'un manque d'autonomie respectivement aux syntagmes prépositionnels (SP) et le type d'information qu'il véhicule est étroitement lié à la présence d'un clitique référentiellement et fonctionnellement identique. En effet, en l'absence de reprise, l'objet n'est plus détaché mais fait partie intégrante de l'énoncé et passe du rôle de support à celui d'apport informationnel. En position postverbale (SVO), il occupe la position focale par défaut (position finale et accent principal) et constitue à lui seul l'apport informationnel de l'énoncé ou en est l'ultime élément et en position préverbale (OVS), l'objet porte l'information assertée de l'énoncé, il est étroitement focalisé (position initiale et accent principal) et l'énoncé répond à un besoin d'identification ${ }^{8}$.

A partir de ce que nous venons d'exposer, voici un résumé des principales caractéristiques syntaxiques de la dislocation en italien (pour un inventaire plus étayé, cf. Cinque, $1991: 283)$ :

1) Position extra-propositionnelle

2) Antéposition et accentuation (DG) ou postposition et désaccentuation (DD)

8 Cette focalisation à l'initiale est largement décrite par les linguistes, sous des dénominations distinctes : «topicalisation rhématique (Stammerjohann, 1986), «topicalisation contrastive » (Renzi, Salvi et Cardinaletti, 1988), « rhématisation à gauche» (Berretta, 1995) ou encore « focus-background structure » (Brunetti, 2009). Nous renvoyons à un article que nous avons consacré à la focalisation en italien (Augendre, 2009 : 189-191) pour plus de détails sur cette structure et sur le besoin communicatif auquel elle répond. 
3) Maintien des marques fonctionnelles

4) Fonction assumée par le pronom clitique (si présent)

5) Coréférence syntagme/pronom et double marquage rectionnel

6) Pas de marquage syntaxique pour un sujet disloqué

7) Dislocation du COD dépendante de la présence d'une reprise et dislocation d'un SP indépendante de la présence d'une reprise

8) Pause/séparation favorisée dans le cas d'un COD disloqué et non favorisée dans le cas d'un SP disloqué

\subsubsection{Caractéristiques informationnelles}

Afin de fournir une explication aux différences syntaxiques et prosodiques décrites précédemment, il est nécessaire de prendre en considération la composante pragmatique et donc de se demander à quels besoins communicatifs peuvent répondre les énoncés avec dislocation. En effet, c'est le but de la prise de parole (pragmatique) qui influe sur l'aspect formel de l'énoncé (syntaxe), qui demande à son tour un schéma accentuel et intonatif cohérent (prosodie).

La dislocation est traditionnellement définie comme le moyen syntaxique par excellence de signaler le thème d'une phrase. On trouve par exemple cette idée chez Simone (1990: 386) ou Larsson (1979:10), qui écrit que le but de la DG est de :

Marquer ouvertement le thème de la phrase [...] le locuteur invite celui à qui il s'adresse à actualiser et à isoler un certain concept, et le prépare ainsi à la communication suivante.

Cependant, plus que le thème, il nous semble que la structure répond au besoin communicatif de marquer certains éléments comme appartenant à l'arrière-plan. De ce point de vue, un élément DG serait un topique, c'est-à-dire une information présentée comme présupposée mais proéminente (détachement à l'initiale et accentuation) et un élément DD un «tail $»^{9}$, autrement dit une information présentée comme 


\section{S. AUGENDRE}

présupposée mais non proéminente (détachement en finale et inaccentuation). C'est dans cette optique que se place par exemple Prévost (2003: 107), qui écrit que dans une DG, l'élément est souvent un "pré-topique » ou un topique «en construction » alors que dans une DD, le même élément est plutôt la «continuation d'un topique déjà établi comme tel ». Prenons deux exemples :

(5a) A Piero, Maria non gli ha detto niente.

« À Pierre, Marie ne lui a rien dit. »

Ici, l'élément DG «a Piero / à Pierre » ainsi que le sujet «Maria/Marie » sont marqués comme appartenant à l'arrièreplan de l'énoncé alors que le SV véhicule l'apport informationnel («n'avoir RIEN dit»). Ajoutons simplement qu'être marqué comme un élément présupposé ne signifie pas être nécessairement donné contextuellement, comme nous le verrons avec les données du corpus. Quoi qu'il est soit, au sein de l'énoncé 5.a, il est clair que «il costituente dislocato è il centro d'interesse del parlante nel proferire la frase $»^{10}$ (Berruto, $1985: 70$ ).

(5b) Maria non gli ha detto niente, a Piero.

« Marie ne lui a rien dit, à Pierre. »

Ici, le syntagme disloqué n'est pas non plus à considérer comme un élément informatif. C'est au sujet «Maria/Marie » que se réfère la prise de parole (topique), le SV porte l'information ajoutée (focus) et le SP DD n'est qu'un ajout ou rappel marqué par la syntaxe, la topologie et la prosodie comme non saillant et déjà actif au niveau contextuel. Ceci dit, parmi les occurrences du corpus, nous verrons qu'il y a aussi des cas où l'élément DD est contextuellement nouveau.

La dislocation servirait donc à marquer le statut communicatif d'un constituant non focalisé : DG, il serait un élément d'arrière-plan saillant alors que $\mathrm{DD}$, il serait un élément d'arrière-plan non saillant. De cela résulterait le fait qu'un énoncé avec DG aurait plus une fonction d'ouverture

10 Trad. : «Le constituant disloqué est le centre d'intérêt du locuteur dans la production de la phrase ». 
conversationnelle et qu'un énoncé avec DD serait plus lié à un phénomène de clôture conversationnelle, hypothèse à vérifier dans la partie suivante.

\section{Le corpus écrit et son analyse}

Notre travail s'appuie sur un corpus exclusivement écrit et relevant de l'informalité, les occurrences de dislocation (200 au total) étant tirées de conversations dialogiques réalisées par le biais de messageries instantanées, e-mail et SMS. En fonction des propriétés formelles et informationnelles des occurrences de DG et DD du corpus, nous allons à présent tenter de vérifier ou d'infirmer les hypothèses que nous avons émises en première partie.

\subsection{Classification des occurrences et statistiques}

A partir des données du corpus, nous avons choisi de distinguer trois types de dislocations en fonction de l'élément concerné par la structure :

1) DG et DD du Sujet (S)

2) $D G$ et $D G$ du COD

3) DG et DD du SP (COI, CC et C. du nom)

Voici les pourcentages obtenus par chacune de ces structures :

\begin{tabular}{|c|c|c|c|}
\hline $\begin{array}{c}\text { Élément } \\
\text { disloqué }\end{array}$ & DG & DD & Total \\
\hline S & $45 \%$ & $7,5 \%$ & $52,5 \%$ \\
\hline COD & $30,5 \%$ & $6,5 \%$ & $37 \%$ \\
\hline SP & $7,5 \%$ & $3 \%$ & $10,5 \%$ \\
\hline Total & $83 \%$ & $17 \%$ & $100 \%$ \\
\hline
\end{tabular}

$\mathrm{Au}$ vu de ce tableau récapitulatif, la première observation qui s'impose est la large domination de la DG (83\% des cas de dislocation) sur la DD (17\% des cas de dislocation) quel que soit le type d'élément disloqué. Ce résultat va dans le sens des études sur corpus déjà réalisées. 


\section{S. AUGENDRE}

En revanche, contrairement aux résultats obtenus dans des corpus oraux ${ }^{11}$, il se trouve qu'ici, ce n'est pas la dislocation du COD (37 \%) mais celle du S (52,5\%) qui domine largement en termes de taux d'utilisation. Comme nous allons le voir par la suite, l'utilisation massive de la DG du S est intimement liée à l'aspect dialogique des conversations du corpus.

Dans cet article, nous nous limiterons à l'analyse des occurrences de dislocation du sujet et de l'objet direct, qui représentent près de $90 \%$ des cas de dislocation du corpus. Audelà de ce critère quantitatif, qui permet certaines généralisations, cette restriction est motivée par la volonté de détailler l'analyse au maximum mais aussi de considérer en parallèle deux arguments similaires d'un point de vue formel, puisqu'ils sont tous deux introduits directement.

\subsection{Propriétés formelles et informationnelles des occurrences}

Nous avons choisi de présenter notre analyse en deux parties, chacune dédiée à la dislocation d'un argument direct. Les résultats seront résumés sous forme de tableaux puis analysés et illustrés par des exemples tirés du corpus.

$\mathrm{Au}$ niveau formel, nous allons analyser les occurrences à partir des cinq points présentés ci-dessous :

1) La reprise (présence $v s$ absence)

2) La pause (marquée vs non marquée)

3) La catégorie de l'élément (pronom vs syntagme)

4) La modalité de l'énoncé (assertion vs interrogation)

5) Le type de proposition d'accueil (principale vs subordonnée)

$\mathrm{Au}$ niveau informationnel, il s'agira de regarder en détail les contextes d'utilisation des occurrences, le statut communicatif de l'élément disloqué et, si nécessaire, celui des autres éléments de l'énoncé.

\subsubsection{Les cas de dislocation du sujet}

Dans notre corpus, ce sont les cas de DS et plus particulièrement de SDG qui dominent largement sur l'ensemble des

11 Dans le corpus de Berruto (1985), 2/3 des cas de dislocation sont des dislocations de l'objet. 
occurrences de dislocation. Comme nous l'avons indiqué précédemment, il nous semble que l'explication de cette utilisation massive réside presque exclusivement dans l'aspect dialogique omniprésent du corpus.

Voici le tableau récapitulatif des principales caractéristiques observées :

\begin{tabular}{|l|c|c|}
\cline { 2 - 3 } \multicolumn{1}{c|}{} & S DG (45 \%) & S DD (7,5 \%) \\
\hline 1) Pause/Séparation & $83 / 90$ & $9 / 15$ \\
\hline 2) Catégorie & 70 pronoms & $11 \mathrm{SN}$ \\
& $20 \mathrm{SN}$ & 4 pronoms \\
\hline 3) Modalité & 46 assertives & 8 assertives \\
& 44 interrogatives & 7 interrogatives \\
\hline
\end{tabular}

1) Comme pour pallier l'absence de reprise clitique dans le cadre d'une DS en italien, quasiment tous les sujets disloqués (92/105) sont séparés de leur prédicat. Cela dit, la virgule n'est présente que dans peu de cas ( 7 au total) et il en est de même pour l'apparition du sujet et du reste de l'énoncé dans deux messages distincts ( 1 cas unique sur MSN). Le type de césure largement utilisé est l'insertion de matériel linguistique entre sujet et prédicat, allant d'un mot unique à une subordonnée. Voici les principaux cas observés dans le corpus, classés par taux de présence décroissant :

- Dans les phrases interrogatives, le sujet est placé avant le pronom interrogatif et apparaît « hors phrase » $(32 \text { cas })^{12}$ :

(6) E tu (//) cosa mi racconti di bello ?

Et toi qu'est-ce que me racontes(2sg.) de beau?

«Et toi (//) qu'est-ce que tu me racontes de beau ?»

- La séparation est effectuée avec une incise (24 cas), transmettant souvent une information de type temporel (10 cas) :

(7) Io (//) fino a settembre (//) sono sotto contratto.

Moi jusqu'à septembre suis( $1 \mathrm{sg}$.) sous contrat.

«Moi, (//) jusqu'à septembre, (//) je suis sous contrat. »

12 Dans 3 autres cas, le sujet apparaît même dans une interrogative distincte :

E tu ? Hai programmi?

«Et toi ? Tu as des programmes?» 
- Un mot unique sépare le sujet du verbe $(18$ cas $)$ : il s'agit principalement d'adverbes ( $12 \mathrm{cas})$, principalement temporels ( 7 cas, ex. 8a.) ou de conjonctions à valeur oppositive (4 cas, ex. 8b.).

(8a) Io (//) ora (//) mi preparo la cena.

Moi maintenant me prépare $(1 \mathrm{sg}$.$) le dîner.$

«Moi, (//) maintenant (//), je me prépare le dîner. »

(8b) Ma tu (//) invece (//) vai all' aeroporto ?

Mais toi en revanche vas(2sg.) à l'aéroport ?

«Mais toi, en revanche, tu vas à l'aéroport? »

- Un COD (7 cas, ex. 9a.) ou un COI ( 2 cas, ex. 9b.) sert de séparateur :

(9a) Io (//) un'idea (//) l'ho.

Moi une idée la ai(1sg.)

« Moi, une idée, je l'ai.'/ 'Moi, une idée, j'en ai une. »

(9b) Lui (//) alla discussione (//) non mi ha invitata.

Lui à la soutenance nég. me a(3sg.) invitée.

«Lui, à la soutenance, il ne m'a pas invitée. »

- Enfin, le sujet de la principale en est séparé par une subordonnée ( 2 cas, ex. 10a.) et inversement, le sujet de la subordonnée en est séparé par la principale ( 2 cas, ex. 10b.) :

(10a) Io (//) se fossi in te (//) non sarei così sicura.

Moi si étais $(1 \mathrm{sg}$.) en toi nég. serais(1sg.) si sûre

« Moi, si j'étais toi, je ne serais pas si sûre. »

(10b) Tu (//) è ormai da un po' (//) che hai iniziato.

Toi est(3sg.) déjà depuis longtemps que as(2sg.) commencé.

« Toi, ça fait déjà un moment que tu as commencé. »

Il apparaît donc clairement que plus qu'une pause (marquée par la virgule à l'écrit), c'est une réelle séparation qu'utilisent les locuteurs afin de garantir au sujet un statut privilégié de topique discursif, qu'il soit nouveau ou réactualisé.

2) En ce qui concerne la catégorie du terme disloqué, il est intéressant de noter que la DG et la DD du S proposent deux tendances opposées. Si pour la DG, les sujets sont presque 
exclusivement des pronoms personnels (70 pronoms contre $20 \mathrm{SN}$ ), pour la DD, la majorité des sujets sont des SN (11 SN contre 4 pronoms). Cette différence nous semble étroitement liée à la position occupée par les sujets : en première position (DG), ces derniers sont immédiatement accessibles et servent à recadrer le discours sur un référent précis alors qu'en dernière position (DD), la conjugaison du verbe fournit déjà des informations sur le sujet, qui est ensuite marqué comme présupposé.

Le lien entre le nombre de cas de DG d'un sujet pronominal et l'aspect dialogique des conversations du corpus semble évident dès que l'on regarde de plus près les types de pronoms disloqués : 32 sont des «tu / tu», 30 sont des « io / je », 5 sont des «noi / nous », et seuls 3 sont des « lei / elle» et « lui / il », renvoyant à des personnes extérieures à la conversation en cours. La fonction de recadrage du discours sur un ou les participants de la discussion semble donc indéniable dans une DG du sujet.

3) Enfin, en termes de modalité, l'équilibre entre assertions (54/105) et interrogatives (51/105) va dans le sens de ce que nous venons de voir concernant les pronoms. En effet, dans le dialogisme, c'est essentiellement par le biais du questionnement que la conversation en cours est (re)cadrée sur un/les interlocuteur/s ou sur un/des référent/s de discours.

4) Pour terminer sur la DS, nous allons mettre en lien les caractéristiques formelles des occurrences avec leurs propriétés communicatives :

- Sur l'ensemble des cas de DG du S, le sujet est une reprise du topique en cours dans seulement 5 cas ( 2 pronoms et $3 \mathrm{SN})$ :

\footnotetext{
A : Ci sono ancora le opere sulle porte ? Y sont(3pl.) encore les oeuvres sur les portes?

«Il y a encore les œuvres sur les portes?»

B : Le opere ancora ci sono.

Les œuvres encore y sont(3pl.).

«Les œuvres, elles y sont encore. »
} 
Dans les 84 cas restants ( 68 pronoms et $16 \mathrm{SN}^{13}$ ), en revanche, le sujet ouvre sur une nouvelle thématique discursive (relance conversationnelle) :

- Quand le sujet disloqué est un SN, son référent est, dans la majorité des cas, introduit pour la première fois dans la conversation en cours (14/16). Dans les 2 cas restants, le référent a déjà été activé puis vient réactualisé. Ces données, ajoutées au fait que 11 des 16 occurrences se trouvent dans des interrogatives, permettent d'établir un lien entre la DG d'un SN sujet et l'introduction d'un nouveau topique et référent de discours. 4 occurrences de «noi / nous » renvoient à un référent de discours inactif (un groupe dont fait partie le locuteur) et sont, de ce point de vue, à rapprocher des cas de SN :

$$
\begin{aligned}
\text { A : } & \text { Mancava l'accento } \\
& \text { Manquait(3sg.) l'accent } \\
& \text { «Il manquait l'accent» }
\end{aligned}
$$

B : Noi italiani non li guardiamo mai quelli !

Nous italiens nég. les regardons jamais ceux-là

« Nous, italiens, nous ne les regardons jamais ceux-là ! »

- Dans les 64 cas restants, tous des pronoms, il s'agit plus d'un recadrage de la conversation sur une ou des personnes déjà présentes dans l'univers de discours. En toute logique, c'est dans cette dernière catégorie que se classent toutes les occurrences de « io / je » et de «tu / tu » DG ainsi que les 3/4 des phrases interrogatives présentes. Précisons également que dans plus de $2 / 3$ des cas (42/64), le sujet disloqué a une valeur dite «oppositive»: «tu / toi » est explicitement opposé à «io / moi » dans 28 cas (ex. 13) et à une personne extérieure dans 1 cas, « io / moi » est clairement opposé à « tu / toi » dans 9 cas (cf. ex. 10a.) et à une personne extérieure dans 4 cas :

13 Nous avons exclu une occurrence de SN disloqué de l'analyse car le sujet introduit un nouveau référent de discours mais ce dernier n'est pas topique :

Anche a Susanna le cose sembra vadano un po' meglio.

Egalement à Susanne les choses semble( $3 \mathrm{sg}$.) aillent un peu mieux.

« Pour Susanne aussi, il semble que les chosent aillent un peu mieux. » 
(13) Prima ho scritto « mi sa che non sei l'unica (alcolizzata)» (//) tu poi hai scritto $(/ /)$ « non ne abbiamo parlato »

D'abord ai(1sg.) écrit me sait(3sg.) que nég. es(2sg.) la seule (alcoolique) toi après as $(2 \mathrm{sg}$.) écrit nég. en avons parlé

«D'abord, j' ai écrit “il me semble que tu n'es pas la seule (alcoolique)", toi, après, tu as écrit "nous n'en avons pas parlé”. »

- La fonction communicative de la DD du S semble dépendre de la modalité de l'énoncé : dans une phrase assertive (8/15), elle tend à clore une thématique conversationnelle, $a$ contrario, dans une interrogative (7/15), elle tend à en ouvrir une nouvelle. Dans le premier cas, le référent du sujet est majoritairement (6/8) la reprise du topique en cours, autrement dit un «tail» (ex. 14a.), alors que dans le second, son référent n'est pas un topique préétabli (7/7) et n'a pas été activé linguistiquement $(5 / 7$, ex. $14 \mathrm{~b}$.), les deux cas de sujets pronominaux mis à part :

(14a) A : Non era per me il messaggio ?

Nég. était(3sg.) pour moi le message

«Il n'était pas pour moi le message ?»

B : Sì era per te il messaggio!

Si était(3sg.) pour toi le message

« Si, il était pour toi le message ! »

(14b) (La connexion de B va et vient)

A : Funziona ancora male internet?

Fonctionne encore mal Internet

«Il fonctionne encore mal Internet? »

La première remarque à tirer des occurrences de DS concerne le sujet pronominal: syntaxiquement optionnel en italien, sa présence massive parmi les cas de DS montre bien à quel point sa réalisation est pragmatiquement contrôlée. Ensuite, au vu de l'analyse des DS du corpus, il nous semble que les notions de nouveau topique et de relance conversationnelle précédemment associées à la DG soient plus que vérifiées pour ce qui est du premier argument. En revanche, les données montrent que 
celles de rappel et de clôture conversationnelle précédemment associées à la DD valent dans le cadre d'un énoncé assertif, mais dans celui de l'interrogation. En lien avec cette modalité, qui amène intrinsèquement à relancer la conversation, la DD d'un sujet fonctionne comme sa DG et permet d'introduire un nouveau topique et référent de discours.

\subsubsection{Les cas de dislocation du COD}

Passons à présent à l'analyse des occurrences de DG du COD du corpus :

\begin{tabular}{|c|c|c|}
\cline { 2 - 3 } \multicolumn{1}{c|}{} & $\begin{array}{c}\text { COD DG } \\
(\mathbf{3 0 , 5} \%)\end{array}$ & $\begin{array}{c}\text { COD DD } \\
(\mathbf{6 , 5} \%)\end{array}$ \\
\hline 1) Reprise & $60 / 61$ & $13 / 13$ \\
\hline 2) Pause/Séparation & $5 / 61$ & $0 / 13$ \\
\hline \multirow{3}{*}{ 3) Catégorie } & $51 \mathrm{SN}$ & 6 subordonnées \\
& 7 démonstratifs & 4 SN \\
& 2 subordonnées \\
1 Sadj $^{14}$ & 3 pronoms \\
\hline 4) Modalité & 50 assertives & 8 assertives \\
& 11 interrogatives & 5 interrogatives \\
\hline
\end{tabular}

1) En ce qui concerne la présence d'une reprise pronominale, nos données confirment celles déjà fournies par les linguistes, la reprise n'étant absente que dans un seul cas de dislocation du $\mathrm{COD}^{15}$. En revanche, pour ce qui est de la présence généralement postulée d'une pause, les occurrences de notre corpus montrent qu'elle est loin d'être nécessaire et utilisée (seuls 5 des COD disloqués sont séparés du reste de l'énoncé par une virgule, du matériel linguistique ou réalisés dans un message distinct) :

14 Voici l'occurrence en question :

Fortissima in matematica, come complimento, lo avevo già detto?

Très forte en mathématiques, comme compliment, le avais(1sg.) déjà dit «Très forte en mathématiques, comme compliment, je l'avais déjà dit? »

15 Dans l'énoncé en question, le COD n'est pas un topique (cf. 1.2.2) : Almeno qualcosina evito di chiedere a mia madre.

Au moins un petit quelque chose j'évite de demander à ma mère

« Au moins, j'évite de demander un petit quelque chose à ma mère. » 
(15) Gli esami invece (//) li ho il 27 e 28.

Les examens en revanche les ai $(1 \mathrm{sg}$.) le 27 et 28

«Les examens, en revanche, (//) je les ai les 27 et 28. »

Contrairement à la DS, la dislocation du COD à l'écrit est donc uniquement marquée par la reprise, pas par l'isolation du syntagme.

2) Au niveau du type d'éléments COD disloqué, la grande majorité sont des SN (55/74), le reste étant composé de démonstratifs $(9 / 74)$ tous anaphoriques sauf un et de subordonnées $(8 / 74)^{16}$.

3) Contrairement aux données concernant la DG du S, les DG du COD apparaissent très majoritairement dans les phrases assertives (50/61). En termes de réalisation, les particularités observées pour le COD sont la présence de DG à l'intérieur de subordonnées (4 cas, ex. 16) ainsi que les cas de DD de subordonnées ( 6 cas avec «lo sai/vedi che.../ tu le sais / vois que... », ex. 17) :

(16) Hai visto [che qualcosina riesco a farla anche io]

$\mathrm{As}(2 \mathrm{sg}$.) vu que quelque chose réussis( $1 \mathrm{sg}$.) à faire la aussi moi « $\mathrm{Tu}$ as vu [qu'un petit quelque chose, je réussis à le faire moi aussi]. »

(17) Lo sai [che mi ha scritto Jake]!

Le sais(2sg.) que me a écrit Jake

«Tu le sais [que Jake m'a écrit] !

Dans l'exemple 17, la subordonnée DD ne contient que des éléments contextuellement nouveaux et remplit la double fonction d'ouvrir une nouvelle conversation et de véhiculer l'information assertée de l'énoncé, donnée qui montre que, comme la modalité de l'énoncé, la catégorie de l'élément disloqué influe sur la visée communicative de la DD.

4) Terminons par les propriétés informationnelles des COD disloqués :

- Sur les 61 COD DG du corpus, 22 sont des topiques «maintenus », parmi lesquels 17 sont réalisés sous forme de

16 Les deux cas restants sont un Sadj DG et un pronom DD. 


\section{S. AUGENDRE}

$\mathrm{SN}$ (ex. 18a.) et 5 sous forme de démonstratifs anaphoriques (ex. 18b.) :

(18a) A : Non dimenticare il cane !

Nég. Oublier le chien

«N'oublie pas le chien! »

B : Il cane l'ho lasciato a casa che dormiva.

Le chien le ai $(1 \mathrm{sg}$.) laissé à maison que dormait $(3 \mathrm{sg}$.)

« Le chien, je l'ai laissé à la maison car il dormait. »

(18b) A : (Ho ricevuto) Una mail di Claudio.

(J'ai reçu) une mail de Claude

«(J'ai reçu) un mail de Claude. »

B : Quella l'ho avuta anche io.

Celle-là la ai(1sg.) eue aussi moi

«Celui-là, je l'ai eu moi aussi. »

Les 39 autres COD DG sont de nouveaux topiques discursifs : 3 renvoient à un référent de discours déjà actif (ex. 19) et 36 à un référent de discours inactif (absent du contexte linguistique précédent $)^{17}$.

(19) Ho deciso...pasta panna e funghi (i funghi li mangia mi auguro).

Ai(1sg.) décidé pâtes crème et champignons (les champignons les mange( $3 \mathrm{sg}$.) me souhaite $(1 \mathrm{sg}$.)

« J'ai décidé... pâtes, crème et champignons (les champignons, il les mange j'espère). »

Lors de l'analyse des occurrences de DG (et de DD) du S, nous avons établi un lien entre modalité interrogative et introduction d'un nouveau référent et/ou topique de discours. Les données relatives à la DG du COD conduisent au même rapprochement : 10 des 11 énoncés interrogatifs contenant une DG de l'objet introduisent un nouveau référent et topique de discours.

Par ailleurs, il est intéressant de noter qu'il existe un lien particulier entre la DG du COD et la réalisation du

1730 sont des nouveaux topiques, 3 des sous topiques et les 3 derniers cas des reformulations ou qualifications du topique en cours. 
deuxième argument direct. Dans 7 cas du corpus, sujet et COD sont tous deux DG dans cet ordre (thématisation des arguments 1 et 2 et focalisation du SV) :

(20a) Io (//) la macchina (//) la uso pochissimo.

Moi la voiture la utilise $(1 \mathrm{sg}$.) très peu

« Moi, la voiture, je l'utilise très peu. »

(20b) $\mathrm{Tu}(/ /)$ questo (//) non l'hai frequentato.

Toi celui-ci nég. le as(2sg.) suivi

« Toi, celui-ci, tu ne l'as pas suivi. »

Dans 44 cas de DG du COD du corpus, le sujet n'est pas réalisé et dans les 10 cas restants, le sujet est focalisé en position finale (thématisation de l'argument 2 et focalisation de l'argument 1) :

(21a) Il questionario devi farlo tu.

Le questionnaire dois( $2 \mathrm{sg}$.) faire le toi

«Le questionnaire, c'est toi qui dois le faire. »

(21b) La vodka la porto io.

La vodka la porte moi

«La vodka c'est moi qui l'amène. »

- Au vu des données du corpus, le statut d'un COD DD semble dépendre, comme celui d'un sujet DD, de la modalité de l'énoncé, mais aussi de la catégorie de l'objet (SN ou pronom vs subordonnée). Parmi les $7 \mathrm{SN}$ et pronoms COD disloqués du corpus, 5 sont des «tails» (ex. 22a.) et 2, présents dans des interrogatives, sont de nouveaux topiques (ex. 22b.) :

(22a) A : E come si chiama la tunisina ? «Et comment s'appelle la tunisienne?»

B : Amina

A : Non la conosco lei

Nég. la connais $(1 \mathrm{sg}$.) elle

« Je ne la connais pas, elle. »

(22b) A : Devo ricaricare tutte le emoticon.

Dois(1sg.) retélécharger toutes les émoticônes

« Je dois retélécharger toutes les émoticônes. » 


\section{S. AUGENDRE}

B : Aspetta, te ne do.

Attends(2sg.) te en donne(1sg.)

«Attends, je t'en donne. »

$[\ldots]$

A : Ce l'hai quella della bambina?

La as(2sg.) celle de la fillette ?

«Tu l'as celle de la fillette?»

Dans les 6 cas restants, l'objet est une subordonnée (cf. ex. 17) dont le statut est à la fois topical et focal : elle constitue un changement de topique discursif mais contient aussi l'apport informationnel de l'énoncé. Dans ces cas, l'élément DD serait, en quelque sorte un « topique focalisé ». Les données relatives à la modalité interrogative et aux subordonnées disloquées montrent que la fonction de la DD n'est pas limitée à celle de rappel lexical mais que cette structure permet également d'ouvrir une thématique conversationnelle.

L'analyse des occurrences de dislocation de l'objet du corpus nous conduit au concept de variabilité, que ce soit au niveau de la catégorie de l'élément disloqué, de sa distribution dans l'énoncé ou encore du statut communicatif de son référent. Les données montrent que la dislocation concerne tous les types de COD ( $\mathrm{SN}$, pronom personnel, démonstratif ou subordonnée), que l'argument dispose d'une grande liberté positionnelle au sein de l'énoncé (dans une principale, dans une subordonnée, hors de la proposition où se trouve le verbe de rattachement) tout en restant fortement ancré dans l'énoncé (reprise et absence de séparation) et qu'au niveau informationnel, la DG et la DD de l'objet peuvent toutes les deux répondre à des besoins communicatifs distincts. Quand il est DG, l'objet oscille entre le statut de nouveau topique discursif (relance conversationnelle) et celui de reprise du topique préétabli (maintien de la thématique conversationnelle en cours). Quand il est DD, son rôle n'est pas limité à celui de rappel lexical et les occurrences du corpus nous ont permis d'identifier au moins deux cas dans lesquels l'objet est introduit en tant que nouvelle thématique conversationnelle : quand le COD est une subordonnée et quand l'énoncé est interrogatif. 
Nous avons également observé que la DG de l'objet est très souvent associée à la non réalisation du sujet, ou, mais de façon beaucoup moins fréquente, à la focalisation et à la dislocation du premier argument. Enfin, l'analyse des cas de DG et de DD de l'objet nous a permis d'établir, comme nous l'avions fait pour la DS, un lien entre dislocation, modalité interrogative et introduction d'un référent de discours inactif comme nouveau topique discursif.

\section{Conclusion}

Ce travail sur corpus écrit a eu pour but d'étudier la dislocation des arguments directs en italien. L'analyse a tout d'abord porté sur l'aspect formel des occurrences (reprise clitique, séparation/ pause entre l'élément disloqué et le reste de l'énoncé, distribution du syntagme disloqué dans l'énoncé), sur la catégorie des éléments disloqués et sur la modalité des énoncés d'accueil (assertion vs interrogation). En lien avec les données précédentes, nous avons ensuite considéré les contextes d'utilisation de la structure et de ses deux variantes (DG et DD) ainsi que la fonction communicative du syntagme disloqué.

Cette analyse a tout d'abord montré la nécessité de dépasser les propriétés généralement attribuées à la DG et à la DD au profit d'une étude au cas par cas afin de fournir une description pertinente. Cependant, même là, il n'est pas possible de tout expliquer et il n'y a parfois que le lien entretenu entre le locuteur et le référent de discours, indépendamment des données contextuelles et des autres participants, qui permettrait de rendre compte du choix entre l'une ou l'autre des deux structures.

Au-delà de ces difficultés, ce travail nous a tout de même permis d'observer certaines régularités. D'un point de vue formel, nous avons noté, entre autres, l'omniprésence d'une pause/séparation dans une DS, celle d'une reprise clitique dans une D du COD, la présence massive de pronoms sujets et de SN objets DG, la diversité catégorielle et positionnelle d'un objet disloqué ou encore l'existence d'un lien entre la DG de l'objet et la réalisation du sujet. Au niveau informationnel, nous avons vu que la DG du S a pour principale fonction celle de centrage thématique et de relance conversationnelle alors que la DG de 


\section{S. AUGENDRE}

l'objet permet à la fois d'introduire un nouveau topique de discours ou d'assurer la continuité thématique de la conversation en cours. En ce qui concerne la DD des deux arguments, nous avons principalement observé l'influence de la modalité de l'énoncé sur la fonction pragmatique de la structure : si les fonctions de clôture conversationnelle et de «tail» respectivement attribuées à la DD et au syntagme disloqué se vérifient globalement pour les énoncés assertifs (sauf cas des subordonnées objets), ce n'est pas le cas pour les interrogatifs. Dans ce type d'énoncés, qui vise intrinsèquement à la relance conversationnelle, la DG et la DD des arguments directs sont étroitement liées à l'introduction d'un nouveau topique, qui, bien souvent, est également un nouveau référent de discours.

Les résultats obtenus nous offrent des données relativement fiables que ce soit en termes de catégorie, de réalisation, de distribution et de statut communicatif du constituant disloqué, ou en termes de modalité, de particularité syntaxique et de rôle discursif des énoncés d'accueil et/ou de leurs constituants. Cependant, la principale conclusion à tirer de ce travail reste l'impossibilité d'analyser la dislocation comme un "phénomène » unique, ni d'un point de vue formel, ni d'un point de vue informationnel.

\section{Références bibliographiques}

Augendre S. (2008). « $\mathrm{S}+\mathrm{V}+\mathrm{O}$ : Ordres marqués et non marqués en italien », Discours 3, [en ligne] http://discours.revues.org/index4653.html

Augendre S. (2009). «La focalisation contrastive en italien: une étude de cas », Suvremena Lingvistika 68 : 183-206.

Berretta M. (1995). «Ordini marcati dei constutuenti maggiori di frase: una rassegna », Linguistica e filologia 1 : 125-170.

Berruto G. (1985). " "Dislocazioni a sinistra" e "grammatica" dell'italiano parlato», in F. De Bellis \& L.-M. Savoia (éd.), Sintassi e morfologia della lingua italiana d'uso. Teorie e applicazioni descrittive. Roma : Bulzoni, 59-82.

Blasco-Dulbecco M. (1999). Les dislocations en français contemporain. Etude syntaxique. Paris : Champion. 
Bonvino E. (2005). Le sujet postverbal. Une étude sur l'italien parlé. Paris : Ophrys.

Brunetti L. (2009). «Discourse functions of fronted foci in Italian and Spanish », in A. Dufter \& D. Jacob (éd.), Focus and Background in Romance Languages. Amsterdam / Philadelphia : Benjamins, 43-81.

Cinque G. (1991). Teoria linguistica e sintassi italiana. Bologna : Il Mulino.

De Mauro T., Mancini F., Vedovelli M., Voghera M. (1993). Lessico di frequenza dell'italiano parlato. Milano: EtasLibri.

Duranti A., Ochs E. (1979). " "La pipa la fumi?". Uno studio sulla dislocazione a sinistra nelle conversazioni », Studi di grammatica italiana $8: 269-301$.

Ferrari A. (2003). Le ragioni del testo. Aspetti morfosintattici $e$ interpuntivi dell'italiano contemporaneo. Firenze: Accademia della Crusca.

Frascarelli M. (2003). «Topicalizzazione e ripresa clitica. Analisi sincronica, confronto diacronico e considerazioni tipologiche », in N. Maraschio \& T. Poggi Salani (éd.), Italia linguistica anno Mille - Italia linguistica anno Duemila. Roma : Bulzoni, 547-562.

Gerdes K., Kahane S. (2006). «L'amas verbal au cœur d'une modélisation topologique du français », Linguisticae investigationes $29,1: 75-89$.

Graffi G. (1994). Le strutture del linguaggio. Sintassi. Bologna : Il Mulino.

Larsson E. (1979). La dislocation en français : étude de syntaxe générative. Lund : LiberLaromedel Gleerup.

Prévost S. (2003). « Détachement et topicalisation: des niveaux d'analyse différents », Cahiers de praxématique 40 : 97-126.

Renzi L., Salvi G., Cardinaletti A. (1988). Grande grammatica italiana di Consultazione. La frase. I sintagmi nominale e preposizionale. Bologna: Il Mulino.

Rossi F. (2005). " Tratti pragmatici e prosodici della dislocazione a destra nel parlato spontaneo », in E. Burr. (a cura 


\section{S. AUGENDRE}

di), Tradizione e innovazione, Il parlato : teoria - corpora - linguistica dei corpora. Firenze : Franco Cesati Editore, 307-321.

Simone R. (1990). Fondamenti di linguistica. Roma / Bari : Laterza.

Sobrero A.A. (1993). "Pragmatica », in A.A. Sobrero (éd.), Introduzione all'italiano contemporaneo. Le strutture. Roma-Bari : Laterza, 403-450.

Stammerjohann H. (1986). Tema e rema in italiano/thema and rhema in Italian. Tübingen : Narr.

Tonfoni G. (1983). La frase semplice e complessa nell'italiano. Parma : Zari.

Vallduví E. (1992). The informational component. New-York : Garland. 